\title{
A Possible Solution for the Cosmological Constant Problem
}

Nick Gorkavyi*i

SSAI

E-mail: gorkavyiegist.us

\author{
Alexander Vasilkov \\ SSAI
}

John Mather

GSFC/NASA

The mysterious acceleration of the expansion of the Universe, discovered in 1998, can be described by the cosmological constant introduced by Einstein in 1917. In the quantum cosmology, the existence of a repulsive force is associated with "dark energy" - a hypothetical substance with a negative pressure. But numerous attempts to obtain from the quantum theory the observed value of the cosmological constant lead to values of 120 orders of magnitude larger than the observed value. In the bouncing cosmology, a collapse of the Universe of the previous cycle is supposed, and then its new divergence. We consider the bouncing cosmology in the light of the latest discovery of gravitational waves and a large number of massive black holes, which are a probable candidate for the role of dark matter. When the Universe collapses, a significant part of the gravitational mass of the merging black holes turns into gravitational waves. During mergers, the size of black holes increases. We call the largest of them the Big Black Hole (BBH). In the stage of expansion, the frequency of mergers of black holes will decrease sharply and the reverse process will begin due to the absorption of gravitational waves: the slow growth of the mass of black holes, first of all the $\mathrm{BBH}$, which has the maximum surface. If we assume that gravitational radiation themselves do not generate a gravitational field, then it can be shown that a sharp decrease in the gravitational mass of the Universe causes strong antigravity, which can be responsible for the Big Bang. The gravitational mass of BBH at the stage of expansion of the Universe will grow and the gravitational influence of BBH will stretch a field of galaxies. This stretching effect is described by the appearance of a cosmological constant in the Friedmann equations. An estimated value of the cosmological constant is in good agreement with the observations. A cyclic model of the Universe can be developed on the basis of periodic transformation of the mass of merging black holes into gravitational waves and absorption of the background gravitational radiation by the BBH.

2nd World Summit: Exploring the Dark Side of the Universe

25-29 June, 2018

University of Antilles, Pointe-Ãă-Pitre, Guadeloupe, France

${ }^{*}$ Speaker. 


\section{Big cosmological questions}

We know the universe we have today: distant galaxies receding from us, cosmic microwave background radiation [1], the abundances of the elements, with evidence for dark matter and dark energy. We have a relatively simple theory of the expansion that fits the observations very well with about 7 numbers. But there are a few mysteries left, and it's not just about dark matter, dark energy, and quantum gravity. It could be time for some more gedanken experiments.

What if the current universe were to stop expanding, say because the dark energy has an equation of state we haven't recognized, or maybe isn't what we think it is? What would happen to the objects we see today? How would they behave as they are pulled together in some giant crunch? Would the stars be crushed and shredded, to evaporate into clouds of elementary particles, or would they fall into black holes and disappear from view? Would the black holes soak up everything including the dark matter we think we have found? What would limit the collapse, if anything? Would the collapse have sufficient symmetry that the universe would become smooth again, or would it subdivide into separate pieces such as black holes? In other words, could our current universe bounce, if it were to stop expanding and fall back in? What happens to entropy, does it continue to increase, or perhaps do we not know how to calculate the entropy of an infinite system?

Or what sort of prior universe could bounce to produce the one we have? The idea of the bouncing universe is very old, and indeed was behind Robert Dicke's search for the cosmic microwave background radiation. The bounce idea is an interesting alternative to the inflationary story. How could we tell them apart experimentally?

And a related question, what happens to the overall expansion rate as the gravitational mass changes form, interchanging among rest energy, kinetic energy, radiant energy, black holes, and gravitational waves? In particular, is Einstein's formulation connecting curvature to the stressenergy tensor complete, and does gravitational radiation contribute to the stress-energy tensor as well as showing up in the curvature? Gerard't Hooft wrote [2]: "I emphasize that any modification of Einstein's equations into something like $S_{\mu \nu}-(1 / 2) R g_{\mu \nu}=k\left(T_{\mu v(\text { matter })}+t_{\mu v(\text { grav })}\right)$, where $t_{\mu v(\text { grav })}$ would be something like a 'gravitational contribution' to the stress-energy-momentum tensor, is blatantly wrong. Writing such a proposal betrays a complete misunderstanding of what General Relativity is about. The energy and momentum of the gravitational field is completely taken into account by the non-linear parts of the original equation."

Perhaps we need to separate (at least mentally) the zero frequency cosmological curvature terms from the high frequency curvature terms representing propagating gravitational waves. Then we would represent the energy density in the propagating waves as an equivalent source term that would naturally replace the stress-energy terms of the matter fields and black holes as they are transformed. This would not be a change from general relativity, but only a separation of zero or low frequency terms from the high frequency terms. If 't Hooft is completely right, then this must be equivalent to the non-linear parts of the original equation.

For example, when two black holes merge, a pulse of gravitational wave energy propagates outwards, and the mass of the new black hole is less than the mass of the merging partners, at least according to all the general relativity calculations that match the observations quite well. Should we see a sudden radial acceleration as the expanding shell of gravitational radiation energy passes 
us? Similar idea was expressed recently by Philip W. Anderson [3]: "In the recent observations of gravitational radiation from a black hole collision event it has been calculated that the mass of the resulting aggregate is several solar masses... less than the sum of the masses of the original pair... The observable universe is becoming lighter at some unknown rate, depending on how much is being irreversibly radiated away. ...This does not seem to be accounted for in the present cosmology, and may be a part, or even the whole, of the 'dark energy' that is now postulated."

If the Earth suddenly had a reduced mass, we would expect a change in gravitational attraction to it. We have detected the transverse gravitational waves, but what about the monopole term? Much has already been said within the context of general relativity. Do we know how to apply Birkhoff's theorem to this situation? Similarly, and this is probably already known, where does the entropy and information in the black holes go when they merge? And, aside from pure theory, is there any possible experiment that could ever be done, to verify the predictions? Also, do other theories of gravity make different predictions?

\section{Bouncing cosmology and the cosmological constant problem}

Lets try to answer to some questions, which were mentioned in Section 1, for example "What happens to the overall expansion rate as the gravitational mass changes?" According to the bouncing cosmology, the Universe shrinks to a small size (few ly), and then again begins to expand (see Fig. 1a). The accumulated heavy elements are destroyed under high temperature conditions. Gamow believed that the present expansion of the Universe "is simply an "elastic rebound "[4]. Dicke et al. [5] described the transformation of heavy nuclei into baryons: "the ashes of the previous cycle would have been reprocessing back to the hydrogen." Peebles described the scenario of reversing the Universe: "Let us extrapolate the expansion of the universe back to redshift $z \sim 10^{10}$, when the temperature was $T \sim 3 \times 10^{10} \mathrm{~K}$, and the characteristic photon energy was $\sim k T \sim 3 \mathrm{Mev}$. At this epoch, the CBR photons are hard enough to photodissociate complex nuclei, leaving free neutrons and protons" [6]. However the reason for "elastic rebound" or Big Bang remained a mystery.

By the end of the 20th century, the problem of the accelerated expansion of the Universe arised [7,8]. This acceleration is well described by introducing a cosmological constant [9] with a value of $1.1 \times 10^{-56} \mathrm{~cm}^{-2}$ [10], but the nature of this repulsive force remains unclear. Zeldovich first discussed the idea of deriving the cosmological constant from the quantum fluctuations of vacuum [11], but the resulting values exceed the observed value of the cosmological constant by 120 orders [12,13]. The well-known Weinberg review of 1989 [12] referenced about one hundred papers dealing with the problem of the cosmological constant. After the discovery of the acceleration of the expansion of the Universe, many thousands of articles were published [14]. However the vacuum fluctuations may not contribute to the cosmological constant [15]. Frequent assertions that the Casimir effect is an evidence of vacuum fluctuations have recently been questioned [16]. Thus, it is possible that the cosmological constant has a non-quantum origin, and this is another complex problem for the bouncing cosmology or any other cosmological model. The bouncing cosmology returns to cosmological agenda during last years [17]. The number of black holes discovered by LIGO can be sufficient to explain dark matter [18]. If the bulk of the Universe is contained in black holes, then how does the bouncing cosmology change? During the collapse of 


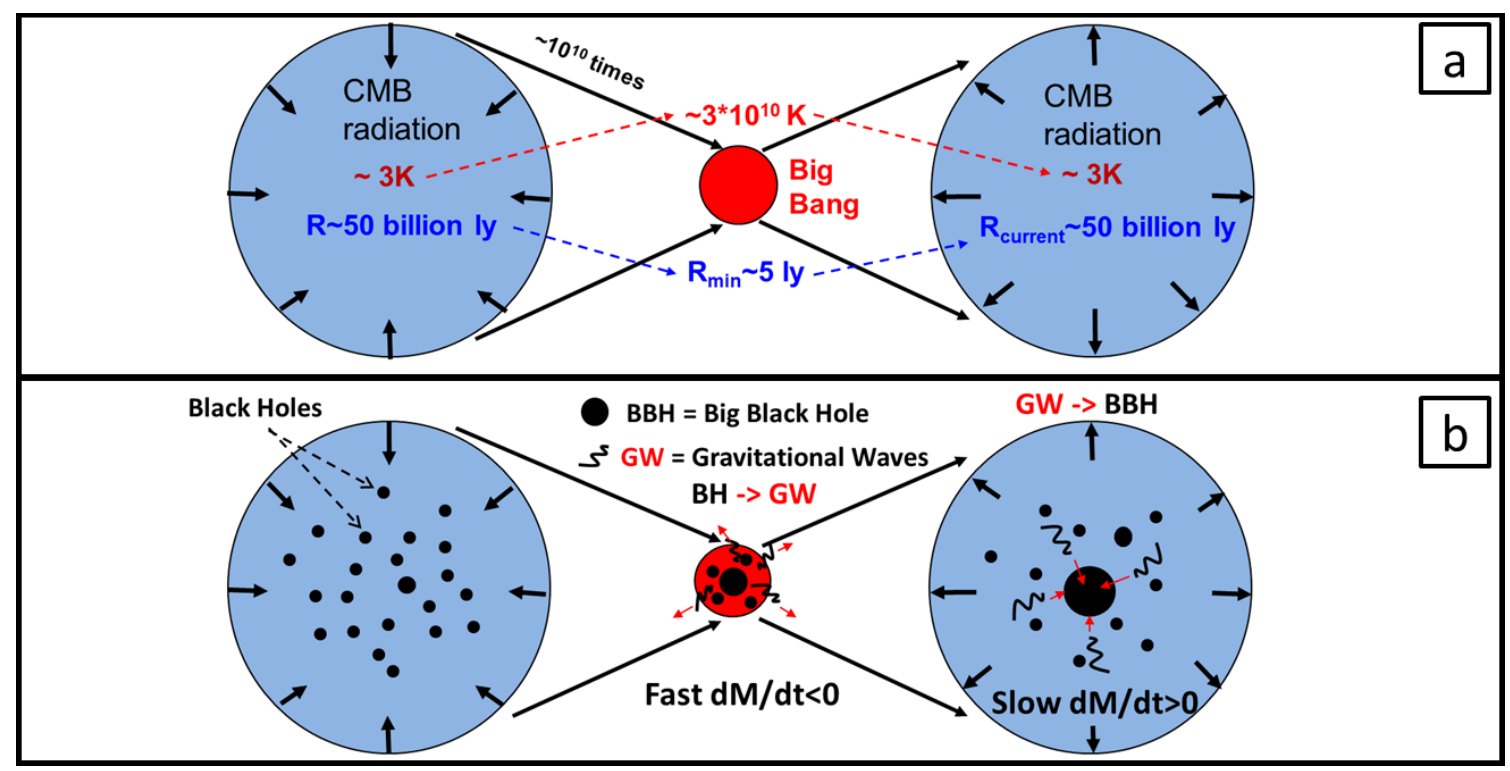

Figure 1: a. Classical bouncing cosmology supposed a reverse and collapse of the Universe to small hot sphere, where a nucleus converted to baryons. b. Modern bouncing cosmology include the black holes as DM. In end of collapse of the Universe, BHs quickly converted to GW; after Big Bang, during expansion, mass of the black holes, including BBH, slowly grow due to absorbing GW.

the Universe the black holes will turn into gravitational waves (see Fig. 1b). With the expansion of the Universe, black holes will grow, absorbing background gravitational waves. The gravitational waves cannot serve as a source of the gravitational field, as Einstein stated [19], but many other scientists do not agree with him [20]. To include or not to include $t_{\mu v(\text { grav })}$ in right side of the Einstein equation? This problem is a part of an axiomatic basis of General Relativity and can not be solved theoretically. This is why we need look at practical consequences from different versions of the answer. In this paper we assume that gravitational radiation does not generate a gravitational field. So, the gravitational mass of the entire Universe will change during mutual transformations the black holes and gravitational waves. We will show that a model of the Universe with a variable gravitational mass can explain both the cause of Big Bang [21] and the origin of the cosmological constant [22].

\section{Explanation of the Big Bang and acceleration of the Universe}

Let us consider a quasi-spherical system comprised of a large number of merging black holes. For a weak gravitational field of a system we have $g_{\mu \nu}=\eta_{\mu \nu}+h_{\mu \nu}$, where $\eta_{\mu \nu}$ is the Minkovsky tensor and $\eta_{\mu \nu}>>h_{\mu \nu}$. We can write the Einstein equation as follows [20,23]:

$$
\square^{2} h_{\mu v}=-\frac{16 \pi G}{c^{4}} S_{\mu v}, \text { where } S_{\mu v} \equiv T_{\mu v}-\frac{1}{2} \eta_{\mu v} T_{\lambda}^{\lambda} .
$$

A solution to Eq. 3.1 is the retarded potentials [20,23]. If we assume that gravitational waves themselves do not generate a gravitational field $[2,19]$, then the zero component of the metric 
tensor [21] is:

$$
g_{00}=-\left[1-\frac{2 G M(t-r / c)}{r c^{2}}\right] .
$$

A similar solution was found by Kutschera [24] for the monopole wave due to changing gravitational mass of relativistic fireballs (see also [11] about possibility of monopole gravitational waves). The metric of Eq. 3.2 is essentially different from the Schwarzschild metric because it describes the varying gravitational mass. Let us describe the diminishing mass of the Universe by an exponential function: $M=M_{0} \exp [-\alpha(t-r / c)]$. Assuming weak gravitational fields and low velocities, we get the following expression for the gravitational acceleration:

$$
a \approx \frac{c^{2}}{2} \frac{\partial g_{00}}{\partial r}=-\frac{G M}{r^{2}}+\frac{\alpha}{c} \frac{G M}{r} .
$$

If $\alpha>0$ the second term of Eq. 3.3 is positive and describes the repulsive force (antigravitation), if $\alpha<0$ the second term describes 'hypergravitation'. To clarify the physical sense of Eq. 3.3 we can rewrite the gravitational acceleration through a quasi-newton potential, $\phi: \quad a=-\frac{\partial \phi}{\partial r}$, where $\phi=-\frac{G M(t-r / c)}{r}$. After differentiation we get expression 3.3. Let us derive modified Friedmann equations for the metric with variable mass in the comoving coordinates $x_{*}, y_{*}, z_{*}$ :

$$
d s^{2}=c^{2} d t^{2}-a^{2}(t, r)[1+b(t, r)]\left(d x_{*}^{2}+d y_{*}^{2}+d z_{*}^{2}\right)
$$

where $b(t, r)=2 G M(t, r) /\left(r c^{2}\right)$ is the known function and $a(t, r)$ is the unknown scale factor. For $|\alpha| \gg c / r$, the dependence of $a(t, r)$ on space coordinates is significantly weaker than that of $b(t, r)$ [22]. The presence of the function $b(t, r)$ in the metric (3.4) results in the additional terms, which can be considered as an effective "cosmological constant". For the case of weak gravitational fields and $b(t, r) \ll 1$, we get the first modified Friedmann equation in the form:

$$
\left(\frac{\dot{a}}{a}\right)^{2}+\left(\frac{\dot{a}}{a}\right) \dot{b}=\frac{\Lambda(t, r) c^{2}}{3}+\frac{8 \pi G \rho}{3}
$$

where the cosmological function $\Lambda(t, r)$ is given by the following expression:

$$
\Lambda(t, r)=\frac{1}{a^{2}}\left(\frac{\partial^{2} b}{\partial x_{*}^{2}}+\frac{\partial^{2} b}{\partial y_{*}^{2}}+\frac{\partial^{2} b}{\partial z_{*}^{2}}\right)=\left(\frac{\partial^{2} b}{\partial x^{2}}+\frac{\partial^{2} b}{\partial y^{2}}+\frac{\partial^{2} b}{\partial z^{2}}\right)
$$

where $x, y, z$ - physical coordinates. From equation 3.6 for $|\alpha| \gg c / r$

$$
\Lambda(t, r) \approx \frac{\alpha^{2}}{c^{2}} b(t, r)=\frac{\alpha^{2}}{c^{2}} \frac{2 G M(t, r)}{r c^{2}}=\frac{\alpha^{2}}{c^{2}} \frac{r_{0}}{r} \approx 0.7 \times 10^{-56}(\alpha T)^{2} \frac{r_{0}}{r}\left[\mathrm{~cm}^{-2}\right]
$$

where $r_{0}$ is the Schwarzschild radius, $T \approx 4 \times 10^{17} \mathrm{sec}$ is the cosmological time. The $\Lambda(t, r)$ is equal to the observed value of the cosmological constant $1.1 \times 10^{-56} \mathrm{~cm}^{-2}$ if $(\alpha T)^{2} \frac{r_{0}}{r}=1.6$ [22]. For example, the latter is valid, if the dimensionless parameters are $\frac{r_{0}}{r}=0.016$ and $|\alpha| T=10$. Let us consider the case when the BBH grows in a medium of relic gravitational radiation with a constant density $\rho_{G W}$. The rate of the BBH mass change is expressed as follows:

$$
\dot{M}=-\alpha M=\rho_{G W} 4 \pi r_{0}^{2} c
$$


Then we get

$$
\rho_{G W}=\frac{|\alpha| c^{3}}{16 \pi G^{2} M}=5 \times 10^{-28} \mathrm{~g} / \mathrm{cm}^{3}
$$

where $|\alpha| T=10$ and the mass of BBH $M=6 \times 10^{54} \mathrm{~g}$ (this mass of BBH can be calculated from the condition $\frac{r_{0}}{r}=0.02$ [22]). The value $\rho_{G W}$ from (3.9) is 25 times greater than the critical density, and approximately two orders of magnitude greater than the average density of matter in the Universe. Let us consider the case when the term with the cosmological function dominates the term with mean density. For an exponential change of the mass of the BBH, we get $\dot{\Lambda}(t, r)=$ $\alpha \Lambda(t, r)$. Then second Friedmann equation:

$$
\frac{\ddot{a}}{a} \approx-\frac{\alpha}{2} \sqrt{\frac{\Lambda(t, r) c^{2}}{3}}
$$

Acceleration of the observed part of the Universe is caused by hypergravity $\alpha<0$ or stretching of a set of galaxies due to inhomogeneous braking or deceleration in the field of growing BBH.

\section{Conclusion}

The cosmology with a variable gravitational mass can explain the cause of the Big Bang, and also leads to a possible solution of the problem of the cosmological constant. From observed value of the modern acceleration of the Universe we can calculate the energy level of the relic gravitational radiation, which is two orders of magnitude greater than the average energy of the matter of the Universe. If the Universe consists of $\sim 99 \%$ of relic gravitational waves, and $\sim 1 \%$ of black holes (as a dark matter), baryons and other elementary particles, then we can put forward a cyclic model of the Universe without introducing hypothetical physical substances or fields. The Big Bang is caused by antigravity, which occurs after the collapse of the Universe and the transition of black holes to gravitational radiation [21]. The Big Black Hole, which survived after the Big Bang, grows due to the absorption of relic gravitational waves. This growth provides the modern accelerated stretching of the field of galaxies, which is characterized by the cosmological constant [22]. A key evidence for this model would be the discovery of high-frequency gravitational waves, which contain the bulk of the energy of the Universe.

\section{Author contributions}

J.M. wrote the section 1 of this article, N.G. and A.V. - other sections.

\section{Acknowledgments}

N.G. thanks the organizers of the conference for their support.

\section{References}

[1] Mather J.C. and Boslough J., 2008, The very first light. Basic Books, New York.

[2] 't Hooft G. Strange misconceptions of general relativity. March 2010, http://www.staff.science.uu.nl/ hooft101 
[3] Anderson Ph.W., 2018, Four last "conjectures", arXiv:1804.11186

[4] Gamow G., 1953, The Creation of the Universe. Viking Press.

[5] Dicke R.H. et al., 1965, Astrophys. J., 142, 414

[6] Peebles P.J.E., 1993, Principles of Physical Cosmology. Princeton Univ. Press.

[7] Perlmutter S. et al., 1999, ApJ., 517, 565.

[8] Riess A.G. et al., 1998, Astron.J., 116, 1009.

[9] Einstein A., 1917, Sitzungsber. Preuss. Acad.Wiss., 142-152.

[10] Ade P.A.R.et al., 2016, Astron. Astrophys., 594, A13, 1.

[11] Zeldovich Ya.B., Novikov I.D., 1971, Stars and Relativity. Chicago Press.Univ.

[12] Weinberg S., 1989, Rev. Modern Phys., 61, 1

[13] Rugh S.E., Zinkernagel H., 2002, Studies in History and Philosophy of Modern Physics, pp.663-705.

[14] O’Raifeartaigh C. et al., 2018, Europ. Phys. Journal, H, v.43, iss.1,pp.37-117.

[15] Brodsky S.J., Shrock R., 2011, Proc.Nat.Acad.Sci.108:45-50.

[16] Jaffe R.L., 2005, Phys.Rev. D72, 021301.

[17] Brandenberger R., Peter P., 2017, Found Phys, 47: 797.

[18] Bird S. et al., 2016, Phys. Rev. Lett., 116, 201301.

[19] Einstein A., 1953, The Meaning of Relativity, 4th edn. Princeton Univ. Press, Princeton

[20] Weinberg S., 1972, Gravitation and Cosmology. John Wiley and Sons, N-Y, London.

[21] Gorkavyi N., Vasilkov A., 2016, MNRAS, 461, 2929-2933.

[22] Gorkavyi N., Vasilkov A., 2018, MNRAS, 476, 1384-1389.

[23] Landau L.D., Lifshitz E.M., 1975, The Classical Theory of Fields. Vol. 2 (4th ed.). Butterworth-Heinemann.

[24] Kutschera M., 2003, Mon.Not.R.Astron.Soc., 345, L1. 